\title{
In vitro antibacterial effect of fosfomycin combination therapy against colistin-resistant Klebsiella pneumoniae
}

This article was published in the following Dove Press journal: Infection and Drug Resistance

\section{Wei Yu ${ }^{1,2, *}$ \\ Qixia Luol,* \\ Qingyi Shi' \\ Chen Huang' \\ Xiao Yu' \\ Tianshui Niu' \\ Kai Zhou' \\ Jiajie Zhang ${ }^{2}$ \\ Yonghong Xiao'}

'State Key Laboratory for Diagnosis and Treatment of Infectious Disease, Collaborative Innovation Center for Diagnosis and Treatment of Infectious Diseases, The First Affiliated Hospital, College of Medicine, Zhejiang University, Hangzhou, People's Republic of China; ${ }^{2}$ Department of Infectious Diseases, Zhejiang Provincial People's Hospital, People's Hospital of Hangzhou Medical College, Hangzhou, People's Republic of China

*These authors contributed equally to this work
Correspondence: Yonghong Xiao State Key Laboratory for Diagnosis and Treatment of Infectious Diseases, Collaborative Innovation Center for Diagnosis and Treatment of Infectious Diseases, The First Affiliated Hospital, College of Medicine, Zhejiang University, No 79 QingChun Road, Hangzhou City, Zhejiang Province, People's Republic of China

Tel/fax +86 57I 8723642 I

Email xiaoyonghong@zju.edu.cn
Objectives: Colistin is still a "last-resort" antibiotic used to manage human infections due to multidrug-resistant (MDR) Klebsiella pneumoniae. However, colistin-resistant K. pneumoniae (CR-Kp) isolates emerged a decade ago and had a worldwide distribution. The purpose of this study was to evaluate the genetic data of CR-Kp and identify the antibacterial activity of fosfomycin (FM) alone and in combination with amikacin (AMK) or colistin (COL) against $\mathrm{CR}-\mathrm{Kp}$ in vitro.

Methods: Three clinical CR-Kp isolates from three patients were collected. Whole-genome sequencing and bioinformatics analysis were performed. The Pharmacokinetics Auto Simulation System 400, by simulating human pharmacokinetics in vitro, was employed to simulate FM, AMK, and COL alone and in combination. Different pharmacodynamic parameters were calculated for determining the antimicrobial effect.

Results: Whole-genome sequencing revealed that none of the three isolates contain $\mathrm{mcr}$ gene and that no insertion was found in $\operatorname{pmr} A B$, phoPQ, or $m g r B$ genes. We found the antibacterial activity of AMK alone was more efficient than FM or COL against CR-Kp. The area between the control growth and antibacterial killing curves of FM ( 8 g every 8 hours) combined with AMK ( $15 \mathrm{mg} / \mathrm{kg}$ once daily) was higher than $170 \mathrm{LogCFU} / \mathrm{mL} \cdot \mathrm{h}^{-1}$. In addition, the area between the control growth and antibacterial killing curves of FM ( $8 \mathrm{~g}$ every 8 hours) combined with COL (75,000 IU/kg every12 hours) was higher than that of monotherapies $\left(>100 \mathrm{LogCFU} / \mathrm{mL}^{\prime} \cdot \mathrm{h}^{-1}\right.$ vs $\left.<80 \mathrm{LogCFU} / \mathrm{mL}^{\circ} \mathrm{h}^{-1}\right)$.

Conclusion: FM ( 8 g every 8 hours) combined with AMK ( $15 \mathrm{mg} / \mathrm{kg}$ once daily) was effective at maximizing bacterial killing against CR-Kp.

Keywords: Pharmacokinetics, pharmacodynamics, monotherapy, combination therapy, colistinresistant Klebsiella pneumoniae

\section{Introduction}

The continuing worldwide emergence of multidrug-resistant Enterobacteriaceae, especially carbapenem-resistant and/or tigecycline-resistant isolates, is becoming an urgent public health threat. ${ }^{1,2}$ Polymyxin, one of the last resorts in the treatment of multidrug-resistant Gram-negative bacteria, is an old antibacterial drug that was recently reintroduced in human medicine. Polymyxin E (colistin) is used most widely and interacts with anionic lipopolysaccharide (LPS) molecules and covalently bonds to a fatty acid chain to enhance antimicrobial activity against Gram-negative bacteria. ${ }^{3}$

Klebsiella pneumoniae, a major concern in the clinic, causes a wide range of infections, from urinary tract infections to pneumonia. Highly resistant K. pneumoniae is emerging worldwide and has become an urgent public health threat. Colistin-resistant 
K. pneumoniae (CR-Kp) was reported not only on account of the plasmid-mediated colistin resistance gene - mcr but also due to chromosome mutation of the related genes $m g r B$ or the PmrAB and PhoPQ two-component systems. ${ }^{4-6}$ $\mathrm{MgrB}$ alteration is a common resistance mechanism of colistin among KPC-producing K. pneumoniae (KPC-Kp) ${ }^{5}$ PmrAB and PhoPQ two-component systems, which are associated with lipopolysaccharide (LPS) modification, play a significant regulatory role in colistin resistance among $K$. pneumoniae isolates. ${ }^{6}$

Therapeutic options for multidrug-resistant (MDR) $K$. pneumoniae are extremely limited, a situation made worse by exhaustion of new drug development for anti-infective agents. Combination therapy is gaining increasing attention to combat the MDR $K$. pneumonia due to the synergistic effect and breadth of the antibacterial spectrum. ${ }^{7}$ However, robust evidence of antimicrobial combination therapy used in clinical practice due to $\mathrm{CR}-\mathrm{Kp}$ infections is lacking. Our previous studies indicated that fosfomycin (FM) enhanced the antibacterial activity of imipenem, ertapenem, tigecycline, colistin (COL), and amikacin (AMK) against 136 KPC-Kp strains, and further in vitro pharmacokinetics/pharmacodynamics (PK/ PD) evaluation showed the combination of FM plus AMK or COL tended to show more prominent bactericidal effect and suppressed the emergence of resistance. ${ }^{8,9}$ Hence, in this study, the genetic data of CR-Kp were investigated and human drug metabolism of FM, AMK, and COL alone and in combination against CR-Kp infections were simulated in vitro.

\section{Methods}

\section{Bacterial strains and antibiotic susceptibility test}

Three clinical CR-Kp strains were used in this study, including 2887, 3155, and 18253, recovered from urine, sputum, and blood, respectively, as part of routine hospital laboratory procedure.

The antimicrobial susceptibility testing of isolates was determined using Mueller-Hinton agar dilution for 21 antibiotics according to the Clinical and Laboratory Standards Institute guidelines. ${ }^{10}$ Escherichia coli ATCC 25922 and Pseudomonas aeruginosa ATCC27853 were used as reference isolates.

\section{Whole-genome sequencing (WGS) and data analysis}

WGS was carried out for the three CR-Kp, with further analyses of gene-environment interaction. Genomic DNA was extracted using the QIAamp DNA Mini Kit (Qiagen, Hilden, Germany) and sequenced using HiSeq 2000 (Illumina, San Diego, CA, USA) by constructing $2 \times 125$ bp pair-end libraries. De novo assembly was done using the CLC Workbench v8.0 (QIAGEN). The resistance genes were identified with BLASTn against the ResFinder 2.1 database (https://cge.cbs.

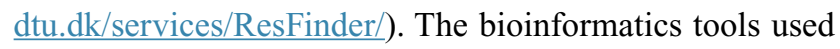
in this study are available on the following web platforms: National Center for Biotechnological Information, Sequence Manipulation Suite, and European Bioinformatics Institute.

This Whole-Genome Shotgun BioProject for the three CR-Kp has been deposited at GenBank under the accession numbers NRJC00000000, NRJD00000000, and NRJE00000000.

\section{Antibiotic dosing}

The human serum concentrations of FM, AMK, and COL were referenced based on previous PK data (Table S1 shows the PK parameters of three drugs and Figure S1 shows the simulated time-concentration curves). ${ }^{1-13}$ The following regimens were simulated over 24 hours: FM 8 g every 8 hours (FM 8 g q8h), ${ }^{11}$ AMK 15 mg/kg once daily (AMK $15 \mathrm{mg} / \mathrm{kg}$ qd), ${ }^{12}$ COL 75,000 IU/kg every 12 hours (COL 75,000 IU/ $\mathrm{kg} \mathrm{q} 12 \mathrm{~h}),{ }^{13} \mathrm{FM}$ (8 g q8h)/AMK (15 mg/kg qd), and FM (8 g q8h)/COL (75,000 IU/kg q12h).

\section{In vitro PK model and PD analysis}

The dynamic model of human PK simulation in vitro was performed using the Pharmacokinetics Auto Simulation System 400 (PASS 400), a product of Dainippon Seiki (Kyoto, Japan) (as shown in Figure S2). The working mechanism and PD parameter calculations were performed as previously described. ${ }^{9}$

A two-compartment model was used for all experiments. Growth curves without antibiotic were regarded as control. There were $1.5 \mathrm{~mL}$ samples collected for CFU determination at predetermined time points $(0,1,2,4,6,8,10,12,14,17,20$, and 24 hours) and diluted with $0.9 \%$ normal saline before plating on Mueller-Hinton agar plates. All counting plates were incubated at $37^{\circ} \mathrm{C}$ overnight. The limit of detection was 30-300 colonies. Each dosing regimen was simulated in triplicate.

A total of 13 PD parameters were calculated, including maximum kill down, maximum kill time, area above kill curve, bacterial growth recovery time (RT), -1Log kill time, $-2 \log$ kill time, $-3 \log$ kill time, regrowth recovery time (SRT), +1Log growth time, Total -1 Log kill time, Total $-2 \log$ kill time, Total $-3 \log$ kill time, and the area between the control growth and antibacterial killing curves (IE). One 
diagram is provided as an illustration to help understand the relationship between different PD parameters (Figure 1). RT and IE were regarded as the most comprehensive parameters for determining the antibacterial activity. ${ }^{14}$

\section{Results}

\section{Antibiotic susceptibility test and drug- resistant characteristics}

Antimicrobial susceptibilities for the three CR-Kp isolates are presented in Table 1. All isolates were resistant to COL with high minimum inhibitory concentration (MIC) level (>32 $\mathrm{mg} / \mathrm{L})$. Isolate 18253 was multidrug resistant. Isolate 2887 was resistant to trimethoprim-sulfamethoxazole and 3155 was resistant to piperacillin-tazobactam, while both isolates were susceptible to other test antibiotics. WGS of these three COLresistant isolates indicated that they all had $\beta$-lactamase genes (Table 1). Isolate 2887 contains $b l a_{\mathrm{SHV}-11}$ and $b l a_{\mathrm{SHV}-75^{\circ}}$. Isolate 3155 contains $b l a_{\mathrm{SHV}-28}$, while isolate 18253 contains $b_{\mathrm{SHV}-11}$, $b l a_{\mathrm{SHV}-12}$, and $b l a_{\mathrm{KPC}-2}$. WGS also revealed that none of the three isolates contained the $m \mathrm{cr}$ gene. In addition, no insertion was found in $p m r A B$, phoPQ, or $m g r B$ genes. Other mechanisms of COL resistance of these isolates are conceivable.

\section{Time-kill antibacterial effect}

The time-kill assays showed that the antibacterial activity of FM/AMK or FM/COL combination was superior to monotherapies against CR-Kp, while regrowth was found as well (Figure 2). AMK monotherapy showed more antibacterial effect than FM and COL. It is of note that FM (8 $\mathrm{g} \mathrm{q} 8 \mathrm{~h})$ alone had little discernible bactericidal effect.

For AMK monotherapy, increasing MIC showed less bactericidal effects against susceptible strains (Figure 2A, C, and E). However, FM/AMK combination enhanced the bactericidal effect, resulting in substantial improvements in bacterial killing (up to $>5 \mathrm{Log} 10 \mathrm{CFU} / \mathrm{mL}$ ) over 24 hours for 2887 (Figure 2A). Also, this combination reduced the population of 3155 and 18253 to $>3 \log 10 \mathrm{CFU} / \mathrm{mL}$ compared with the initial count at 14 hours (Figure $2 \mathrm{C}$ and $\mathrm{E}$ ). Thus, FM/AMK was the most potent combination against both test strains.

FM/COL demonstrated synergistic activity as well, although the three isolates were COL resistant. It is noteworthy that more rapid bacterial killing activity was observed for the combination of FM (8 g q8h)/COL (75,000 IU/kg q12h) against CR-Kp than FM and COL monotherapies (Figure 2B, $\mathrm{D}$, and $\mathrm{F}$ ). FM/COL combination resulted in a reduction in bacterial count $>3 \log 10 \mathrm{CFU} / \mathrm{mL}$ against 3155 at 24 hours.

\section{PD parameters of different dosages against CR-Kp}

The PD parameters were calculated using the built-in Analyze Bactericidal Activity software of PASS 400 (Table 2). In the FM (8 g q8h) monotherapy time-kill assays, RT of 2887 and

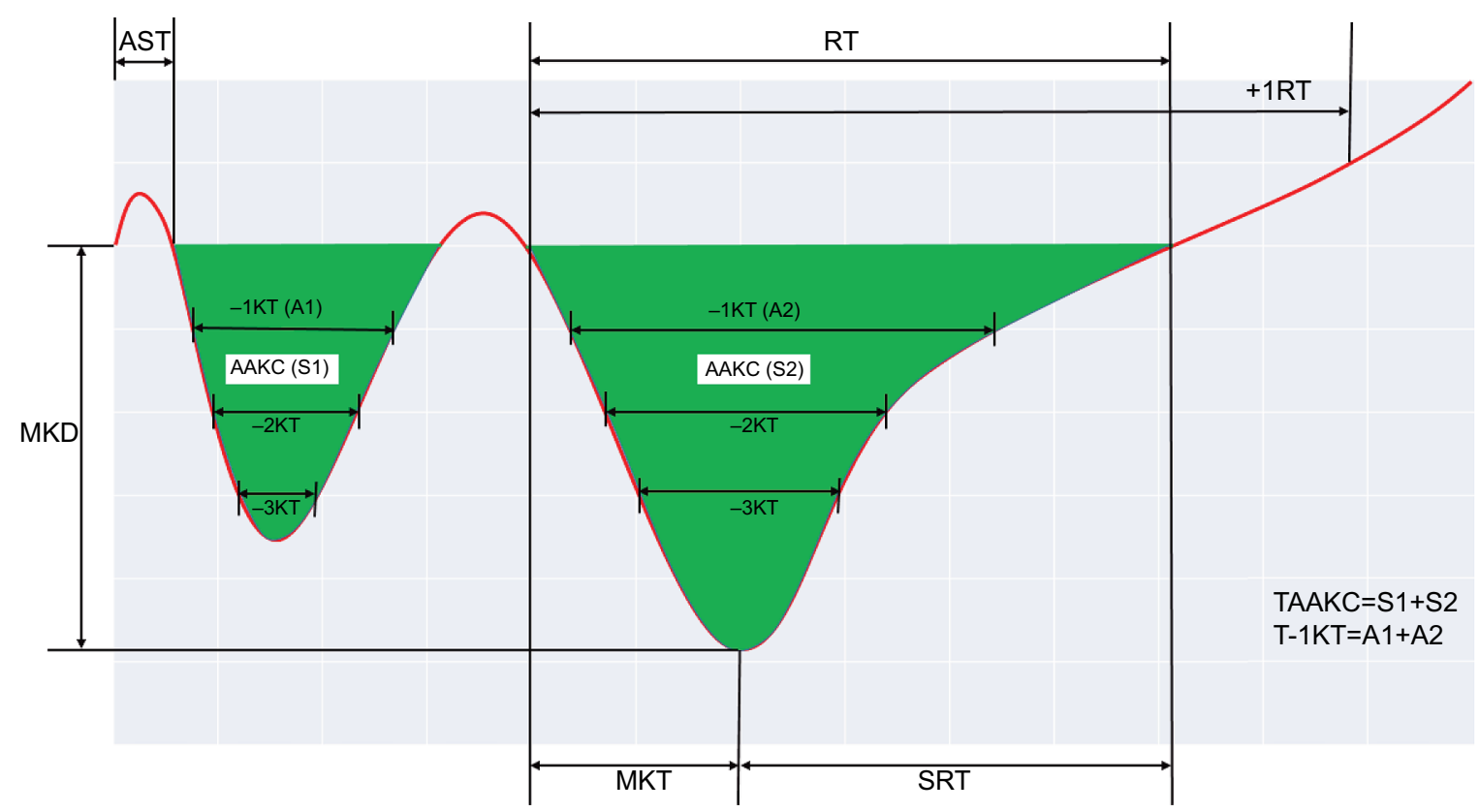

Figure I The sketch of PD parameters.

Abbreviations: -IKT, -I Log kill time; -2KT, -2Log kill time; -3KT, -3Log kill time; SRT, regrowth recovery time; +IRT, +ILog growth time; AAKC, area above kill curve; AST, analysis start time; MKD, maximum kill down; MKT, maximum kill time; PD, pharmacodynamics; RT, bacterial growth recovery time; TAAKC, total area above kill curve; T-IKT, total - ILog kill time. 
Table I Characteristics of three KPC-producing Klebsiella pneumoniae strains

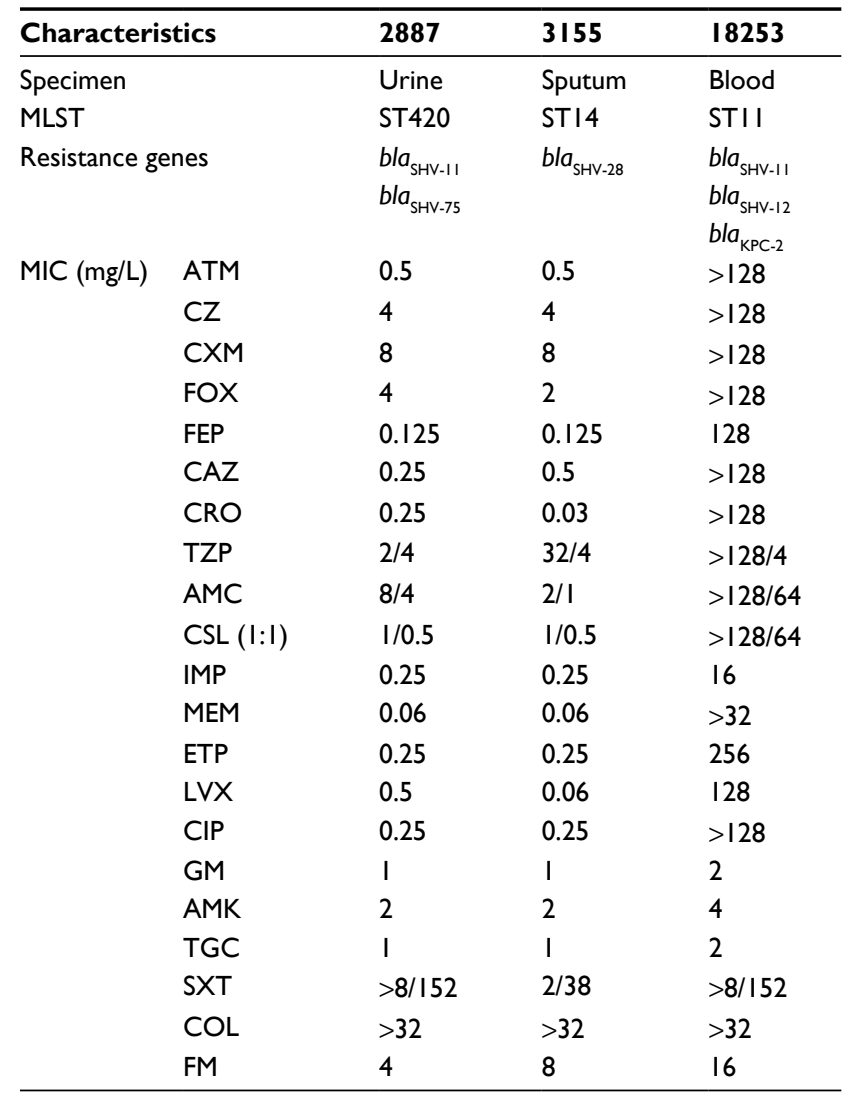

Abbreviations: AMC, amoxicillin-clavulanic acid; AMK, amikacin; ATM, aztreonam; CAZ, ceftazidime; CIP, ciprofloxacin; COL, colistin; CRO, ceftriaxone; CSL, cefoperazone-sulbactam; CXM, cefuroxime; CZ, cefazolin; ETP, ertapenem; FEP, cefepime; FM, fosfomycin; FOX, cefoxitin; GM, gentamicin; IMP, imipenem; LVX, levofloxacin; MEM, meropenem; MIC, minimum inhibitory concentration; MLST, multilocus sequence typing; SXT, trimethoprim-sulfamethoxazole; TGC, tigecycline; TZP, piperacillin-tazobactam.

3155 (>13 hours) was longer than that of 18253 (1.9 hours), and these results may be related to the sensitive status of these three strains. Indeed, AMK alone showed greater bactericidal effect against susceptible strains than FM or COL monotherapies. AMK (15 mg/kg qd) monotherapy showed longer RT ( $>16$ hours) and $-3 \log$ Kill Time T ( $>12$ hours), higher maximum kill down ( $>6 \mathrm{LogCFU} / \mathrm{mL})$, and larger IE ( $>130$ $\left.\operatorname{LogCFU} / \mathrm{mL}^{-1} \mathrm{~h}^{-1}\right)$. Although the three strains were resistant to $\mathrm{COL}$, IE of FM/COL combination was still greater than 100 $\log \mathrm{CFU} / \mathrm{mL} \cdot \mathrm{h}^{-1}$ and RT exceeded 13.8 hours. Importantly, the IEs of FM ( $8 \mathrm{~g} \mathrm{q} 8 \mathrm{~h}) / \mathrm{AMK}(15 \mathrm{mg} / \mathrm{kg} \mathrm{qd})$ against 2887 , 3155 , and 18253 were $219.9,201.1$, and $173.72 \mathrm{LogCFU} /$ $\mathrm{mL} \cdot \mathrm{h}^{-1}$, respectively.

\section{Discussion}

In this study, three CR-Kp with high MIC level ( $>32 \mathrm{mg} / \mathrm{L}$ ) were selected to evaluate the effect of FM, AMK, and COL monotherapy and in combination using an in vitro $\mathrm{PK} / \mathrm{PD}$ simulation model. The findings of this study revealed that FM/AMK or FM/COL resulted in more prominent antibacterial activity against CR-Kp, especially for FM (8 g q8h)/ AMK $(15 \mathrm{mg} / \mathrm{kg}$ qd) combination. FM monotherapy showed little discernible effect. AMK alone achieved more significant bactericidal activity than FM and COL (Figure 2). Of note, the combinations of FM/COL and FM/AMK were more efficient than monotherapies. The reason is that the increase in active uptake of AMK into the cell was caused by FM, resulting in sufficient intracellular concentrations to retard bacterial growth by inhibiting protein synthesis. ${ }^{15}$ In addition, COL may increase penetration of FM to the site of action by permeabilizing the outer bacterial membrane. ${ }^{16}$

$\mathrm{COL}$, belonging to cationic polypeptide antibiotics, is used as a last resort for infections caused by MDR Gramnegative bacteria. Unfortunately, the prevalence of COLresistant bacteria is increasing globally due to its clinical and veterinary uses. ${ }^{17}$ Resistance of bacteria to COL is most commonly related to LPS modifications via several mechanisms involving two-component regulatory systems. ${ }^{6}$ Recently, a plasmid-mediated resistant gene $\mathrm{mcr}$ caused concern owing to the potential spread of COL resistance between humans and animals. ${ }^{4,5}$ However, none of the abovementioned mechanisms have been found in our tested three isolates. Thus, the COL resistance mechanisms of these isolates could be due to other reasons. On the one hand, some other resistance mechanisms of chromosome encoding remain to be identified in CR-Kp. However, it is really difficult to deduce whether some amino acid substitutions found in the clinical isolates are known to be involved in COL resistance. On the other hand, the expression levels of the corresponding genes vary and might consequently influence the resistance to COL in CR-Kp. Since other, novel mechanisms of COL resistance are also conceivable, further investigation is warranted to unveil the mechanism of action.

In order to counteract the COL-resistant strains, recent studies have shifted to studying drugs in combination. As previously reported, $\mathrm{COL}$ alone or in combination in the study showed rapid killing activity during the first hour in the time-kill experiments, but subsequent regrowth was observed in most combinations. ${ }^{18}$ Against COL-susceptible KPC-Kp strains, $\mathrm{FM} / \mathrm{COL}$ combination was shown to be synergistic in chequerboards and time-kill experiments. ${ }^{8} 18$ The same was true for hollow-fiber infection model experiments and the dynamic model of PASS 400 simulation., ${ }^{9}$ Michalopoulos et $\mathrm{a}^{20}$ demonstrated that intravenous administered FM/COL had better bacteriological and clinical outcome for six patients with carbapenem-resistant $K$. pneumoniae. 
A

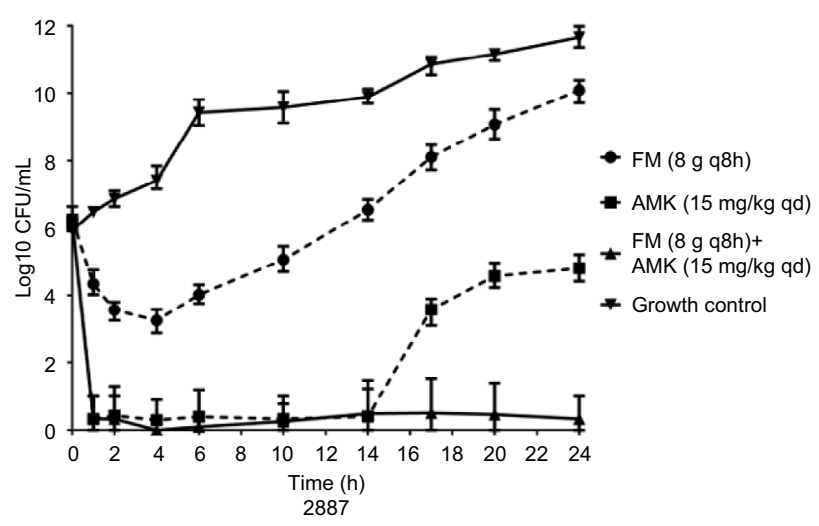

C

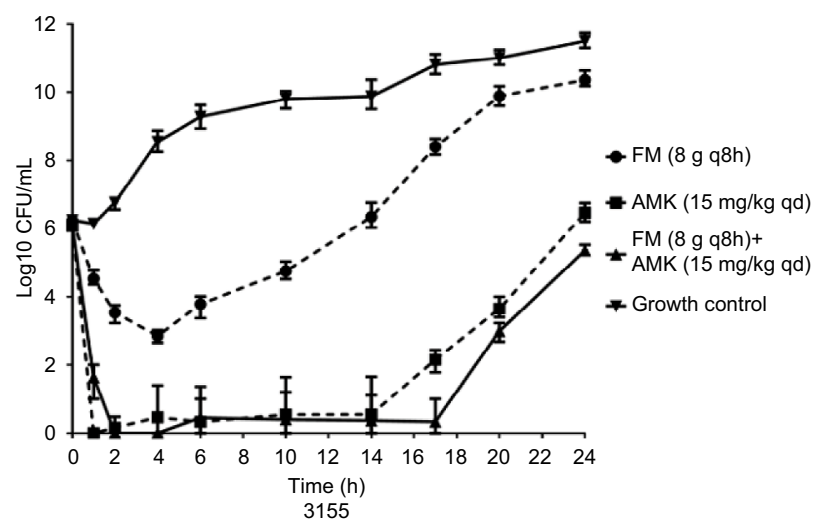

E

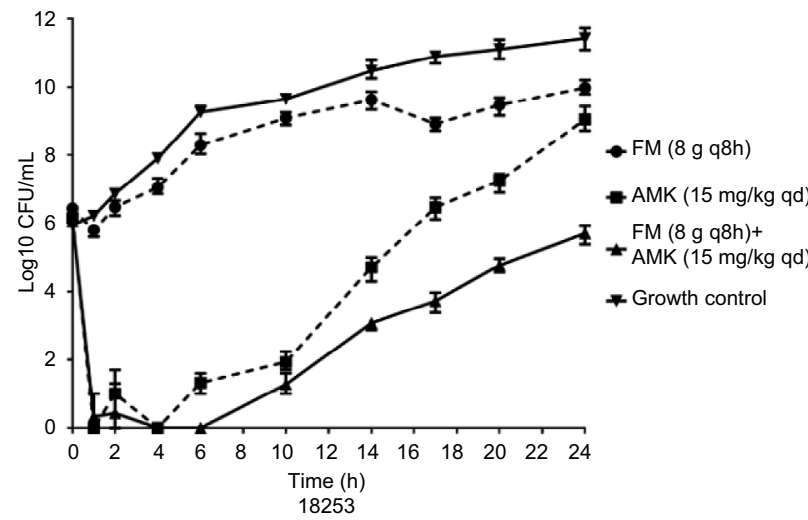

B

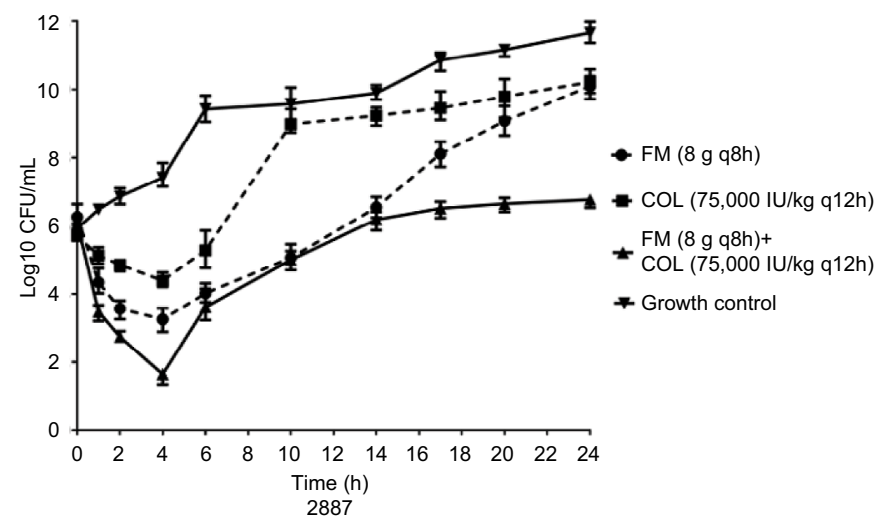

D

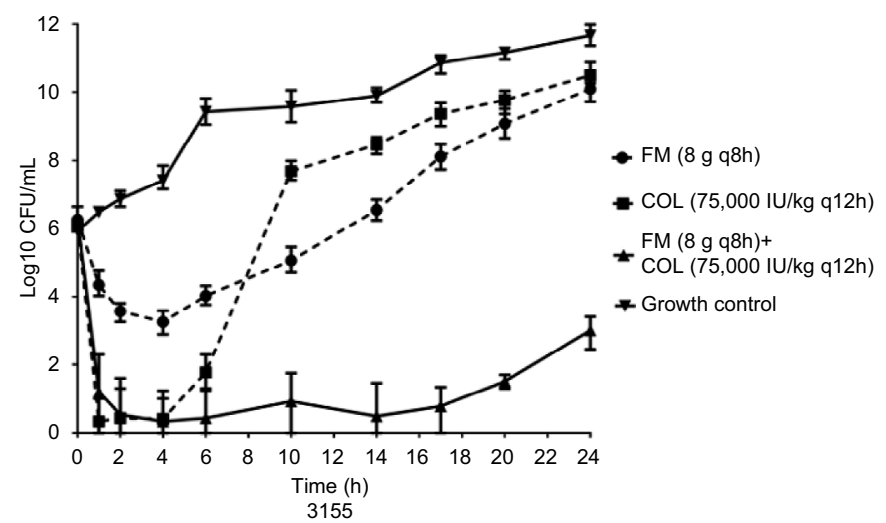

$\mathbf{F}$

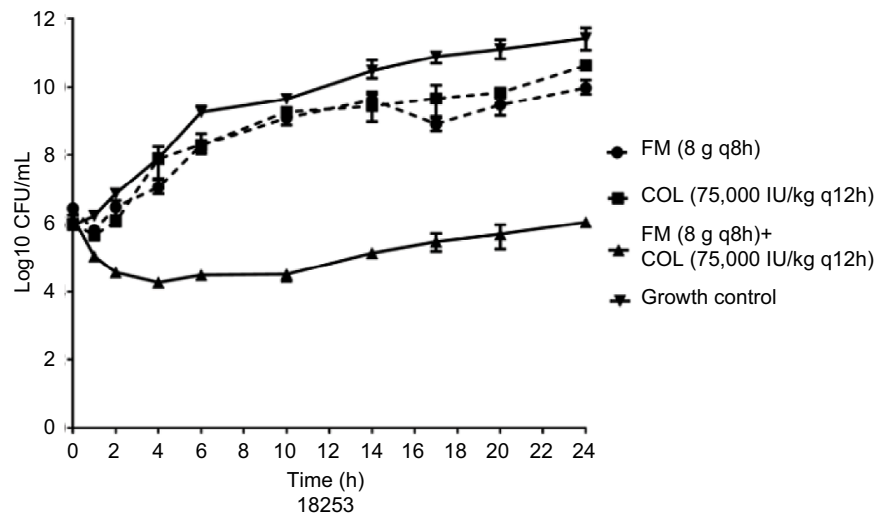

Figure 2 In vitro dynamic model time-kill assays using concentrations of FM, AMK, and COL (either alone or in combination) against three CR-KP strains.

Notes: (A) and (B) monotherapy or combination therapy, respectively, against isolate 2887; (C) and (D) monotherapy or combination therapy, respectively, against isolate 3155; (E) and (F) monotherapy and combination therapy, respectively, against isolate 18253. The dotted lines indicate monotherapy, and the solid lines indicate combination therapy. Antibiotic concentrations are denoted by different symbols.

Abbreviations: AMK, amikacin; COL, colistin; CR-Kp, colistin-resistant Klebsiella pneumoniae; FM, fosfomycin; q8h, every 8 hours; q $12 \mathrm{~h}$, every 12 hours; qd, once daily.

In this study, FM/COL combination showed more rapid bacterial killing than monotherapies, consistent with our previous reports. ${ }^{8,18} \mathrm{FM} / \mathrm{COL}$ combination prolonged the time of antibacterial effect, but unfortunately, regrowth was still present against CR-Kp isolates. Although the IE of FM/ COL combination was still greater than $100 \mathrm{LogCFU} / \mathrm{mL} \mathrm{h}^{-1}$ for CR-Kp isolates, it was still significantly lower compared to COL-susceptible strains $\left(>200 \mathrm{LogCFU} / \mathrm{mL} \cdot \mathrm{h}^{-1}\right){ }^{9}$ This may be associated with the synergistic mechanism of COL permeabilizing the outer bacterial membrane.

In the present study, the bacterial killing of AMK monotherapy at 10 hours was almost similar to FM/AMK 


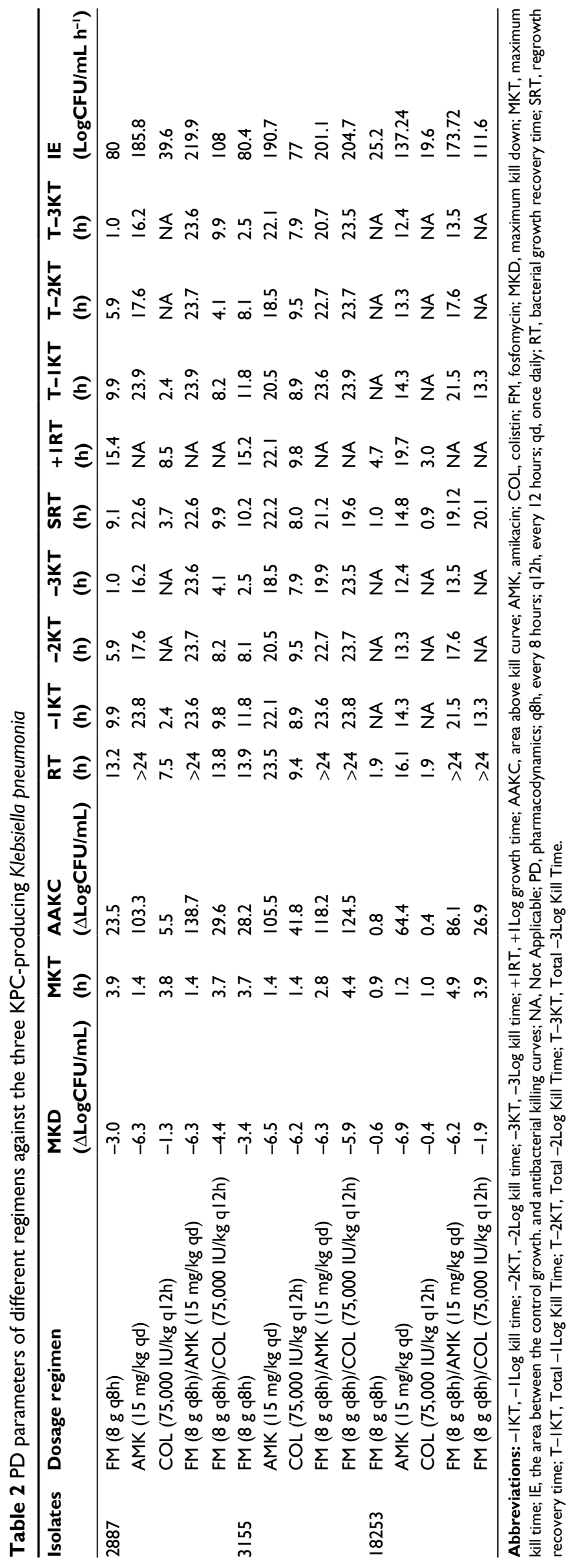

combination; however, the bacterial killing was definitely lower than that of the combination. The IE of FM ( $8 \mathrm{~g} \mathrm{q} 8 \mathrm{~h}) /$ AMK (15 mg/kg qd) against the three CR-Kp strains was much larger $\left(>170 \mathrm{LogCFU} / \mathrm{mL} \cdot \mathrm{h}^{-1}\right)$ than the monotherapies (Table 2). In addition, the studies on activity of FM/AMK combination in vitro showed additive and synergistic effect against MDR Acinetobacter baumannii, P. aeruginosa, and heterogeneous methicillin-resistant Staphylococcus aureus. ${ }^{21-24}$ Sime et a ${ }^{25}$ demonstrated that initial concentrations of $300 \mathrm{mg} / \mathrm{L}$ AMK combined with 1,200 mg/L of FM resulted in rapidly decreasing bacterial burden and preventing the resistant subgroup against $K$. pneumoniae using a dynamic hollow-fiber infection model over 7 days. Notably, FM (8 g q 8 h)/AMK (7.5 mg/kg q12h) combination against KPC-Kp could lead to resistance due to concentration-dependent activity of AMK. ${ }^{9}$ Similarly, better clinical effectiveness was also observed for the combination than with the monotherapies. For example, Vergara-López et $\mathrm{al}^{26}$ demonstrated that prolonged and high dose use of FM combined with AMK was effective in severe systemic infections due to MDR bacteria, with significantly safer profile. Furthermore, a phase 1 study supported the aerosolization of the FM/AMK combination to enable higher tracheal aspirate concentrations, so as to achieve rapid bactericidal effect. ${ }^{27}$ Animal studies have demonstrated that the addition of FM resulted in protective effect for aminoglycoside-related nephrotoxicity by inhibiting aminoglycoside-induced histamine release from mast cell destruction. ${ }^{28}$ To the best of our knowledge, this is the first report on FM/AMK combination against CR-Kp by in vitro simulation. However, there is little known about a randomized double-blind clinical trial of intravenous infusion FM/AMK.

Conjoint analysis of PK/PD could help to maximize treatment efficacy, decrease adverse effects, and minimize resistance rate, especially for the optimized use of antimicrobial agents with different routes of administration and dose. The results of our study suggest that FM/AMK combination is the optimal drug for CR-Kp infections. Further animal experiments and prospective randomized controlled clinical trials need to be done to test the efficacy and reliability of refractory infections.

\section{Conclusion}

In conclusion, the in vitro PK/PD simulation study demonstrated the superior bactericidal activity of FM/COL and FM/ AMK combinations compared with monotherapies against CR-Kp, especially for the combination of FM (8 g q8h)/AMK $(15 \mathrm{mg} / \mathrm{kg} \mathrm{qd})$. These results need to be evaluated in further animal infection models and clinical trials. 


\section{Acknowledgment}

This work was supported by a Key Research and Development Program from Zhejiang Province (No. 2015C03032).

\section{Author contributions}

YHX and WY developed the concept and designed the experiments. WY, QXL, CH, and XY performed the experiments. QXL, TSN, and KZ performed statistical analysis. YHX gave conceptual advice. WY and QXL wrote the paper. All authors discussed the results and implications and commented on the manuscript at all stages. All authors contributed toward data analysis, drafting and critically revising the paper and agree to be accountable for all aspects of the work.

\section{Disclosure}

The authors report no conflicts of interest in this work.

\section{References}

1. Tzouvelekis LS, Markogiannakis A, Psichogiou M, Tassios PT, Daikos GL. Carbapenemases in Klebsiella pneumoniae and other Enterobacteriaceae: an evolving crisis of global dimensions. Clin Microbiol Rev. 2012;25(4):682-707.

2. Spanu T, De Angelis G, Cipriani M, et al. In vivo emergence of tigecycline resistance in multidrug-resistant Klebsiella pneumoniae and Escherichia coli. Antimicrob Agents Chemother. 2012;56(8):4516-4518.

3. Karaiskos I, Souli M, Galani I, Giamarellou H. Colistin: still a lifesaver for the 21st century? Expert Opin Drug Metab Toxicol. 2017;13(1):59-71.

4. Liu YY, Wang Y, Walsh TR, et al. Emergence of plasmid-mediated colistin resistance mechanism MCR-1 in animals and human beings in China: a microbiological and molecular biological study. Lancet Infect Dis. 2015;16(2):161-168.

5. Poirel L, Jayol A, Nordmann P. Polymyxins: antibacterial activity, susceptibility testing, and resistance mechanisms encoded by plasmids or chromosomes. Clin Microbiol Rev. 2017;30(2):557-596.

6. Poirel L, Jayol A, Bontron S, et al. The mgrB gene as a key target for acquired resistance to colistin in Klebsiella pneumoniae. J Antimicrob Chemother. 2015;70(1):75-80.

7. Rafailidis PI, Falagas ME. Options for treating carbapenem-resistant Enterobacteriaceae. Curr Opin Infect Dis. 2014;27(6):479-483.

8. Yu W, Shen $\mathrm{P}, \mathrm{Bao} \mathrm{Z}$, et al. In vitro antibacterial activity of fosfomycin combined with other antimicrobials against KPC-producing Klebsiella pneumoniae. Int J Antimicrob Agents. 2017;50(2):237-241.

9. Yu W, Zhou K, Guo L, et al. In vitro pharmacokinetics/pharmacodynamics evaluation of fosfomycin combined with amikacin or colistin against KPC2-producing Klebsiella pneumoniae. Front Cell Infect Microbiol. 2017;7:246.

10. Clinical and Laboratory Standards Institute. Performance standards for antimicrobial susceptibility testing. 26th informational supplement. Wayne, PA: CLSI; 2016. Available from: http://www.clsi.org/. Accessed January 2016.

11. Sauermann R, Karch R, Langenberger H, et al. Antibiotic abscess penetration: fosfomycin levels measured in pus and simulated concentration-time profiles. Antimicrob Agents Chemother. 2005;49(11): 4448-4454.

12. Garraffo R, Drugeon HB, Dellamonica P, Bernard E, Lapalus P. Determination of optimal dosage regimen for amikacin in healthy volunteers by study of pharmacokinetics and bactericidal activity. Antimicrob Agents Chemother. 1990;34(4):614-621.
13. Mizuyachi K, Hara K, Wakamatsu A, Nohda S, Hirama T. Safety and pharmacokinetic evaluation of intravenous colistin methanesulfonate sodium in Japanese healthy male subjects. Curr Med Res Opin. 2011;27(12):2261-2270.

14. Firsov AA, Vostrov SN, Shevchenko AA, Cornaglia G. Parameters of bacterial killing and regrowth kinetics and antimicrobial effect examined in terms of area under the concentration-time curve relationships: action of ciprofloxacin against Escherichia coli in an in vitro dynamic model. Antimicrob Agents Chemother. 1997;41(6):1281-1287.

15. Cai Y, Fan Y, Wang R, An MM, Liang BB. Synergistic effects of aminoglycosides and fosfomycin on Pseudomonas aeruginosa in vitro and biofilm infections in a rat model. J Antimicrob Chemother. 2009;64(3):563-566.

16. Vaara M. Polymyxins and their novel derivatives. Curr Opin Microbiol. 2010;13(5):574-581.

17. Grégoire N, Aranzana-Climent V, Magréault S, Marchand S, Couet W. Clinical pharmacokinetics and pharmacodynamics of colistin. Clin Pharmacokinet. 2017;56(12):1441-1460.

18. Tängdén T, Hickman RA, Forsberg P, Lagerbäck P, Giske CG, Cars O. Evaluation of double- and triple-antibiotic combinations for VIM- and NDM-producing Klebsiella pneumoniae by in vitro time-kill experiments. Antimicrob Agents Chemother. 2014;58(3):1757-1762.

19. Zhao M, Bulman ZP, Lenhard JR, et al. Pharmacodynamics of colistin and fosfomycin: a 'treasure trove' combination combats KPC-producing Klebsiella pneumoniae. JAntimicrob Chemother. 2017;72(7):1985-1990.

20. Michalopoulos A, Virtzili S, Rafailidis P, Chalevelakis G, Damala M, Falagas ME. Intravenous fosfomycin for the treatment of nosocomial infections caused by carbapenem-resistant Klebsiella pneumoniae in critically ill patients: a prospective evaluation. Clin Microbiol Infect. 2010;16(2):184-186.

21. Martinez-Martinez L, Rodriguez G, Pascual A, SuárezAI, Perea EJ. In-vitro activity of antimicrobial agent combinations against multiresistant $\mathrm{Aci}$ netobacter baumannii. JAntimicrob Chemother. 1996;38(6):1107-1108.

22. Tessier F, Quentin C. In vitro activity of fosfomycin combined with ceftazidime, imipenem, amikacin, and ciprofloxacin against Pseudomonas aeruginosa. Eur J Clin Microbiol Infect Dis. 1997;16(2):159-162.

23. David C, Chaste A, Saison C, Combremont AG. Activité in vitro des associations de la nétilmicine et de l'amikacine avec la fosfomycine et la péfloxacine sur trente et une souches de Staphylococcus aureus méticilline-résistantes hétérogènes. [In vitro effect of combinations of netilmicin and amikacin with fosfomycin and pefloxacin on 31 strains of heterogeneous methicillin-resistant Staphylococcus aureus.] Pathol Biol (Paris). 1988;36(5 Pt 2):608-612. French.

24. O'Hara K, Nakamura A, Shigenobu F, Chen J, Sawai T. [Combination effect of fosfomycin to $\beta$-lactam, aminoglycoside, and macrolide antibiotics against clinical isolates of Klebsiella pneumoniae.] Jpn J Antibiot. 1997;50(8):704-710. Japanese.

25. Sime FB, Johnson A, Whalley S, et al. Pharmacodynamics of aerosolized fosfomycin and amikacin against resistant clinical isolates of Pseudomonas aeruginosa and Klebsiella pneumoniae in a hollow-fiber infection model: experimental basis for combination therapy. Antimicrob Agents Chemother. 2017;61(1):pii: e01763-16.

26. Vergara-López S, Domínguez MC, Conejo MC, Pascual Á, RodríguezBaño J. Prolonged treatment with large doses of fosfomycin plus vancomycin and amikacin in a case of bacteraemia due to methicillin-resistant Staphylococcus epidermidis and IMP-8 metallo- $\beta$-lactamase-producing Klebsiella oxytoca. J Antimicrob Chemother. 2015;70(1):313-315.

27. Montgomery AB, Vallance S, Abuan T, Tservistas M, Davies A. A randomized double-blind placebo-controlled dose-escalation phase 1 study of aerosolized amikacin and fosfomycin delivered via the PARI investigational eFlow $®$ inline nebulizer system in mechanically ventilated patients. J Aerosol Med Pulm Drug Deliv. 2014;27(6):441-448.

28. Bendirdjian JP, Morin JP, Foucher B, Fillastre JP. Effetto della fosfomicina sulla respirazione dei mitocondri renali di ratto. [Effect of fosfomycin on respiration by rat kidney mitochondria.] Minerva Med. 1978;69(59):4079-4086. French. 


\section{Supplementary materials}
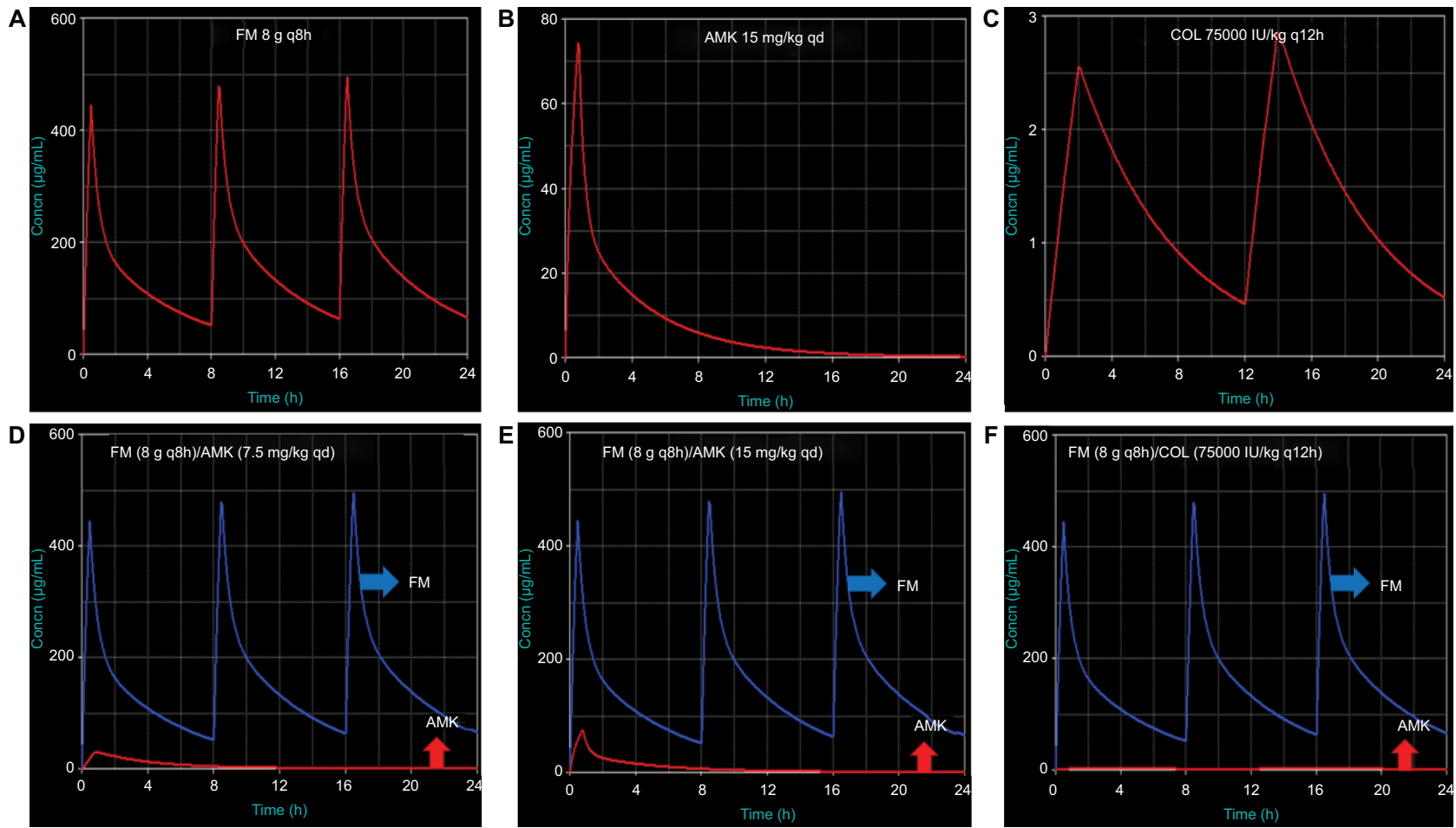

Figure SI (A-F) The time-concentration curves of antibiotics in the Pharmacokinetics Auto Simulation System 400.

Abbreviations: FM, fosfomycin; AMK, amikacin, COL, colistin; q8h, every 8 hours; q $2 \mathrm{~h}$, every 12 hours; qd, once daily.

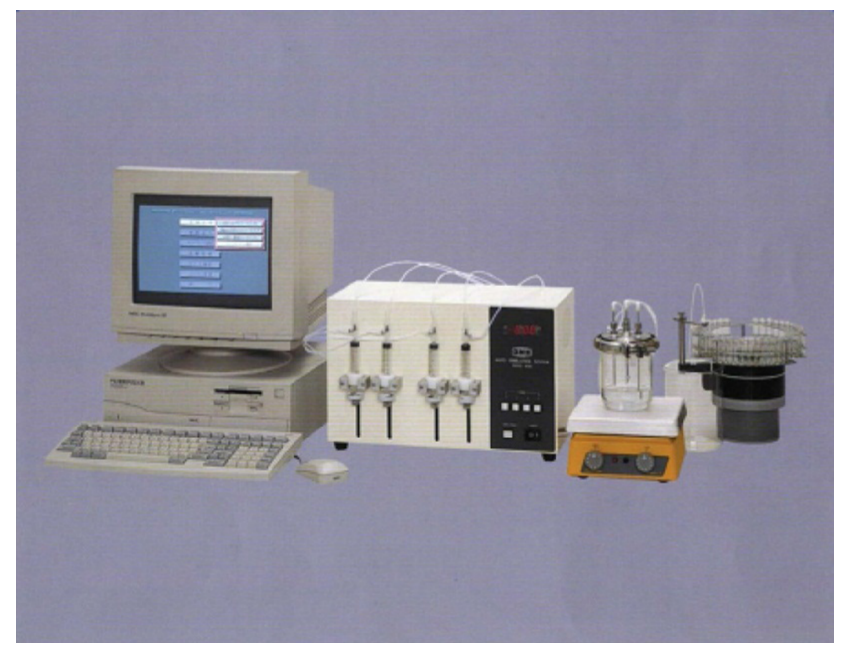

Figure S2 Pharmacokinetics Auto Simulation System 400.

Table SI PK parameters of different regimens

\begin{tabular}{|c|c|c|c|c|c|}
\hline Regimens (11-13) & 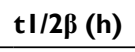 & AUC (mg h/L) & $\mathrm{CL}(\mathrm{mL} / \mathrm{min})$ & $V(L)$ & $C_{\max }(\mathrm{mg} / \mathrm{L})$ \\
\hline AMK $15 \mathrm{mg} / \mathrm{kg}$ once daily & $2.4 \pm 0.5$ & $154.5 \pm 29.9$ & $112.8 \pm 9.2$ & $11.2 \pm 1.8$ & 76 \\
\hline COL $2.5 \mathrm{mg} / \mathrm{kg}$ every 12 hours & $4.0 \pm 0.7$ & $17.6 \pm 6.80$ & $10.5 \pm 4.1$ & $94.9 \pm 30.2$ & 2.55 \\
\hline FM $8 \mathrm{~g}$ every 8 hours & $3.7 \pm 2.2$ & $1,330 \pm 609$ & $126 \pm 68$ & $28.6 \pm 9.9$ & 443.6 \\
\hline
\end{tabular}

Note: Data presented as mean \pm SD.

Abbreviations: AMK, amikacin; AUC, area under the plasma concentration-time curve; $C_{\max }$, maximum plasma concentrations; $\mathrm{CL}$, body clearance; COL, colistin; $\mathrm{FM}$, fosfomycin; $\mathrm{tl} / 2 \beta$, elimination half-life; $\mathrm{PK}$, pharmacokinetics; $V$, volume of distribution. 
Infection and Drug Resistance is an international, peer-reviewed openaccess journal that focuses on the optimal treatment of infection (bacterial, fungal and viral) and the development and institution of preventive strategies to minimize the development and spread of resistance. The journal is specifically concerned with the epidemiology of antibiotic resistance and the mechanisms of resistance development and diffusion in both hospitals and the community. The manuscript management system is completely online and includes a very quick and fair peerreview system, which is all easy to use. Visit http://www.dovepress.com/ testimonials.php to read real quotes from published authors.

Submit your manuscript here: https://www.dovepress.com/infection-and-drug-resistance-journal 